\title{
DERIVATIVES AT THE BOUNDARY FOR ANALYTIC LIPSCHITZ FUNCTIONS
}

\author{
ANTHONY G. O'FARRELL
}

\begin{abstract}
We consider the behaviour of holomorphic functions on a bounded open subset of the plane, satisfying a Lipschitz condition with exponent $\alpha$, with $0<\alpha<1$, in the vicinity of an exceptional boundary point where all such functions exhibit some kind of smoothness. Specifically, we consider the relation between the abstract idea of a bounded point derivation on the algebra of such functions and the classical complex derivative evaluated as a limit of difference quotients. We show that whenever such a bounded point derivation exists at a boundary point $b$, it may be evaluated by taking a limit of classical difference quotients, for approach from a set having full area density at $b$.
\end{abstract}

\section{INTRODUCTION}

In a first paper [11] on the subject, we confined attention to the situation in which the boundary point is nicely accessible from $U$. This paper reports on a continuation of the investigation described in [11], and we refer the reader to that paper for background and notation.

We recall for emphasis that the main object of interest is the algebra $A=A_{\alpha}(U)$ of lip $\alpha$ (note the "little-lip") functions on the bounded open set $U \subset \mathbb{C}$, and that $0<\alpha<1$.

We note one necessary condition that follows from the theorem of [6]: If there exists a nonzero continuous point derivation on $A=A_{\alpha}(U)$ at a given boundary point $b$, then $U$ has full area density at $b$, i.e.

$$
\lim _{r \downarrow 0} \frac{\mathcal{L}^{2}(U \cap \mathbb{B}(b, r))}{\pi r^{2}}=1,
$$

where $\mathbb{B}(b, r)$ denotes the closed ball of radius $r$. Here we use the notation that $\mathcal{L}^{d}$ denotes $d$-dimensional Lebesgue measure.

Date: May 9, 2018.

2010 Mathematics Subject Classification. 30E25, 30H99, 46J10.

Key words and phrases. Analytic function, boundary, Lipschitz condition, point derivation, difference quotient, capacity, Hausdorff content. 
We say that a sequence $\left(z_{n}\right)_{n}$ of points of $U$ converges non-tangentially to $b$, written $z_{n} \rightarrow_{\mathrm{nt}} b$, if there exists a constant $t>0$ such that

$$
\operatorname{dist}\left(z_{n}, \mathbb{C} \backslash U\right) \geq t\left|z_{n}-b\right|, \forall n .
$$

Our main result in [11] was the following:

Theorem 1.1. Let $0<\alpha<1$, let $U \subset \mathbb{C}$ be a bounded open set, let $b \in \operatorname{bdy}(U), z_{n} \in U, z_{n} \rightarrow_{\mathrm{nt}} b$. Suppose $A=A_{\alpha}(U)$ admits a nonzero continuous point derivation at $b$. Let $\partial$ be the normalised derivation at b. Then for each $f \in A$, we have

$$
\frac{f\left(z_{n}\right)-f(b)}{z_{n}-b} \rightarrow \partial f
$$

Now we consider the general situation, in which there may not exist any sequence from $U$ that approaches $b$ nontangentially. Without going into details about Hausdorff content, we remark that for a closed ball $M_{*}^{\beta}(\mathbb{B}(a, r))=r^{\beta}$, and also that $M_{*}^{\beta}$ is countably subadditive. As a result, it is easy to construct many examples $U$ in which the complement of $U$ is a countable union of closed balls, and the singleton $\{b\}$, and in which $A$ has a continuous point derivation at $b$, even though there is no sequence $z_{n} \in U$ approaching $b$ nontangentially. All you have to do is make sure that the sum of the $(1+\alpha)$-th powers of the radii of all the closed balls that meet $A_{n}(b)$ is no greater than $s_{n} / 4^{n}$, where $\sum_{n} s_{n}<+\infty$.

Here is our main result:

Theorem 1.2. Let $0<\alpha<1$, let $U \subset \mathbb{C}$ be a bounded open set, and let $b \in \operatorname{bdy}(U)$. Suppose $A=A_{\alpha}(U)$ admits a nonzero continuous point derivation at $b$. Let $\partial$ be the normalised derivation at $b$. Then there exists a set $E \subset U$, having full area density at $b$, such that for each $f \in A$, we have

$$
\lim _{E \ni z \rightarrow a} \frac{f(z)-f(b)}{z-b}=\partial f .
$$

As with the earlier theorem, we would expect that this result could be extended to higher order derivations and to weak-star continuous derivations on the big Lip algebra.

This result allows us to give a number of conditions equivalent to the existence of a bounded point derivation at $b$ :

1 There is a misprint in Equation (3), page 142 of this paper: $\alpha$ should be $1-\alpha$. Also, on page 144 , line 5 , it should say $R_{a}-L_{a}=(1-\hat{T}(a)) R_{a}$. With these corrected, the argument is valid and the result stands. 
Corollary 1.3. Let $0<\alpha<1$, let $U \subset \mathbb{C}$ be a bounded open set, and let $b \in \operatorname{bdy}(U)$. Then the following four conditions are equivalent:

(1) $A=A_{\alpha}(U)$ admits a nonzero continuous point derivation at $b$.

(2) There exist $a_{n} \in U, a_{n} \rightarrow b$, such that $\frac{f\left(a_{n}\right)-f(b)}{a_{n}-b}$ is bounded for each $f \in A$.

(3) There exist $a_{n} \in U, a_{n} \rightarrow b$, such that $\frac{f\left(a_{n}\right)-f(b)}{a_{n}-b}$ converges for each $f \in A$.

(4) There exists $E \subset U$ having full area density at $b$ such that $\lim _{E \ni a \rightarrow b} \frac{f(a)-f(b)}{a-b}$ exists for each $f \in A$.

In the paper [11] we introduced a number of new ideas that allow the use of arguments similar to those used in the theory of uniform algebras in this present context of Lipschitz algebras with exponent less than 1. Essentially, the main idea is that $\operatorname{lip} \alpha$, for $0<\alpha<1$ is "sufficiently close" to $C^{0}$, so that the elements of its dual space, although they are not measures, are "sufficiently similar" to measures so that methods that work for measures can also be made to work for them. We are going to use these same ideas, supplemented by some observations drawn from potential theory, to prove our main theorem.

The paper is organised as follows: In Section 2 we make a few preliminary remarks and establish that Corollary 1.3 will follow from the main theorem. In Section [3, we establish a few lemmas from potential theory. These real-variable results about Riesz potentials hold not just in two dimensions, and are presented here in the context of $d$ dimensional Euclidean space. In section 4 we recall the tools involving representing measures and the Cauchy transform that were introduced in [11, and we apply the lemmas from potential theory to derive estimates for Cauchy transforms. In Section 5 we complete the proof of the main theorem.

\section{Preliminaries}

2.1. Standing Conventions in this Paper. Throughout the paper, $0<\alpha<1, U \subset \mathbb{C}$ is a bounded open set, $A=A_{\alpha}(U), Y=\operatorname{clos}(U)$, and $b \in X=\operatorname{bdy}(U)$. By measure we shall mean a Borel-regular complex measure $\mu$ such that its total-variation measure is a Radon measure, i.e. assigns finite measure to each compact set.

2.2. Extensions. Let lip $\alpha$ denote, for short, the global $\operatorname{space} \operatorname{lip}(\alpha, \mathbb{C})$ of bounded lip $\alpha$ functions on $\mathbb{C}$. This space forms a Banach algebra under pointwise operations. Each $f \in A$ may be extended (in many 
different ways) to an element of lip $\alpha$, without increasing its pure seminorm $\|f\|^{\prime}=\kappa(f)$ or supremum (cf. [11]). Thus the restriction map to $U$ (or $Y$, or $X$ ) makes $A$ isometric to a quotient algebra of

$$
\tilde{A}=\{f \in \operatorname{lip} \alpha: f \text { is holomorphic on } U\} .
$$

We shall find it convenient to work with globally-defined functions in the sequel.

Let us denote

$$
\mathcal{A}:=\{f \in \tilde{A}: f \text { is holomorphic near } b\} .
$$

Then [6] $\mathcal{A}$ is a dense subalgebra of $\tilde{A}$, and the restriction algebra $\mathcal{A} \mid U$ is a dense subalgebra of $A$. As a consequence, the set of functions $f \in A$ that extend holomorphically to a neighbourhood of $b$ is dense in $A$.

\subsection{Proof of Corollary $\mathbf{1 . 3}$,}

Proof. Theorem 1.2 tells us that condition (1) in the corollary implies condition (4). It is obvious that $(4) \Longrightarrow(3) \Longrightarrow(2)$, so we just have to see that $(2) \Longrightarrow(1)$.

Suppose (2) holds. Then by the Banach-Steinhaus Theorem there is a constant $M>0$ such that

$$
\left|\frac{f\left(a_{n}\right)-f(b)}{a_{n}-b}\right| \leq M\|f\|_{A}
$$

for each $f \in A$. Hence if $f \in A$ extends holomorphically to a neighbourhood of $b$, we have $\left|f^{\prime}(b)\right| \leq M\|f\|_{A}$. Since the set of such functions is dense in $A$, it follows that the functional $f \mapsto f^{\prime}(b)$ has a unique continuous extension $\partial: A \rightarrow \mathbb{C}$, and clearly $\partial$ is a point derivation at $b$.

\section{Tools from Potential Theory}

3.1. The Riesz Capacities. Fix $d \in \mathbb{N}$. Let $0<s<d$. For compact $K \subset \mathbb{R}^{d}$ we define $C_{s}(K)$, the order $s$ Riesz capacity of the set $K$, to be the supremum of the total variations $\|\mu\|$, where $\mu$ ranges over all positive Radon measures that are supported on $K$ and have $\frac{1}{|x|^{s}} * \mu \leq 1$ on $\mathbb{R}^{d}$, i.e. the Riesz potential

$$
\int_{K} \frac{d \mu(x)}{|x-y|^{s}} \leq 1, \forall y \in \mathbb{R}^{d}
$$

For general sets $E \subset \mathbb{R}^{d}$, we then define $C_{s}(E)$ to be the inner capacity

$$
\sup _{K \subset E, K \text { compact }} C_{s}(K) \text {. }
$$


For more about these capacities, see [3, 7]. We note from [3], for future use, the following facts about $C_{s}$ :

(1) $C_{s}(\mathbb{B}(a, r)) \leq r^{s}$ for all $a \in \mathbb{R}^{d}$ and $r>0$.

(2) There is a constant $c$, depending only on $d$, such that $C_{s}(\mathbb{B}(a, r)) \geq c \cdot r^{s}$ for all $a \in \mathbb{R}^{d}$ and $r>0$.

(3) More generally, $C_{s}(E) \geq c \cdot \mathcal{L}^{d}(E)^{\frac{s}{d}}$ for all $E \subset \mathbb{R}^{d}$.

(4) $C_{s}$ is countably subadditive.

3.2. Estimate for the potential. We need a couple of estimates involving these capacities and potentials. The first is the following:

Lemma 3.1. Let $b \in \mathbb{R}^{d}, \mu$ be a positive Borel-regular measure on $\mathbb{R}^{d}$ having compact support and no mass at $b$, let $0<s<d, \epsilon>0$, and

$$
E=\left\{a \in \mathbb{R}^{d}:|a-b|^{s} \cdot \int_{\mathbb{R}^{d}} \frac{d \mu(x)}{|x-a|^{s}} \geq \epsilon\right\} .
$$

Then

$$
\sum_{n=1}^{\infty} 2^{s n} C_{s}\left(A_{n}(b) \cap E\right)<+\infty .
$$

Here, as usual, $A_{n}(b)$ denotes an annulus:

$$
A_{n}(b)=\left\{x \in \mathbb{R}^{d}: \frac{1}{2^{n+1}} \leq|x-b| \leq \frac{1}{2^{n}}\right\} .
$$

Corollary 3.2. Under the same assumptions on $b, \mu, s, \delta$ and $E$, the set $E$ has Lebesgue density zero at b, i.e.

$$
\lim _{r \downarrow 0} \frac{\mathcal{L}^{d}(E \cap \mathbb{B}(b, r))}{r^{d}}=0 .
$$

This lemma is well-known to potential-theorists, and may be found in the book of Mizuta [7, Theorem 5.3]. (One should remark that the case $(d, s)=(2,1)$ of the corollary is due to Browder and was used in the proof (1967) of his Metric Density Theorem [1, Theorem 3.3.9, p.177], and that the same case of the full theorem was known in 1974 [8]. The case $d=2,1<s<2$ was given in [9, page 421]. The case $(d, s)=(d, d-2)$ is in the book of Armitage and Gardiner [4, Theorem 7.7.2].)

The Corollary is immediate from the fact (cited above) that the capacity $C_{s}(E)$ is bounded below by a constant multiple of $\mathcal{L}^{d}(E)^{\frac{s}{d}}$. 
3.3. Estimate for the double-layer potential. The second estimate involves a double-layer potential:

Lemma 3.3. Let $b \in \mathbb{R}^{d}$, $\mu$ be a positive measure on $\mathbb{R}^{d} \times \mathbb{R}^{d}$ having compact support and no mass on $\{b\} \times \mathbb{R}^{d}$ or on $\mathbb{R}^{d} \times\{b\}$, let $s>0$, $t>0,0<s+t<d, \epsilon>0$, and

$$
E=\left\{a \in \mathbb{R}^{d}:|a-b|^{s+t} \cdot \int_{\mathbb{R}^{d} \times \mathbb{R}^{d}} \frac{d \mu(x, y)}{|x-a|^{s}|y-a|^{t}} \geq \epsilon\right\} .
$$

Then

$$
\sum_{n=1}^{\infty} 2^{(s+t) n} C_{s+t}\left(A_{n}(b) \cap E\right)<+\infty .
$$

This seems to be new, and we shall provide a proof.

We remark that the integral is finite for almost all $a$ with respect to area measure, as may be seen by applying Fubini's theorem.

Proof of Lemma 3.3. Suppose that, on the contrary,

$$
\sum_{n=1}^{\infty} 2^{(s+t) n} C_{s+t}\left(A_{n}(b) \cap E\right)=+\infty .
$$

Fix a positive number $M$ greater than the diameter of the support of $\mu$ and greater than the distance from $(b, b)$ to any point of the support of $\mu$.

Fix $N \in \mathbb{N}$. Since $C_{s+t}\left(A_{n}(b) \cap E\right)$ does not exceed $2^{-(s+t) n}$, we may choose $M \geq N$ (depending on $N$ ) such that

$$
2 \leq \sum_{n=N}^{M} 2^{(s+t) n} C_{s+t}\left(A_{n}(b) \cap E\right) \leq 3 .
$$

For each $n$ from $N$ to $M$, choose a positive measure $\nu_{n}$ supported on a compact subset of $A_{n}(b) \cap E$, with $\nu_{n} * \frac{1}{|x|^{s}} \leq 1$ and $\left\|\nu_{n}\right\| \geq$ $\frac{1}{2} \cdot C_{s+t}\left(A_{n}(b) \cap E\right)$. Let $\lambda_{N}:=\sum_{N}^{M} 2^{(s+t) n} \nu_{n}$. Then $\lambda_{N}$ is supported in $\mathbb{B}\left(b, 2^{-N}\right)$ and has $1 \leq\left\|\lambda_{N}\right\| \leq 3$. Also

$$
\begin{aligned}
\delta & \leq \int|a-b|^{s+t} \int \frac{d \mu(x, y)}{|x-a|^{s}|y-a|^{t}} d \lambda_{N}(a) \\
& =\int G_{N}(x, y) d \mu(x, y),
\end{aligned}
$$

where

$$
G_{N}(x, y):=\int \frac{|a-b|^{s+t}}{|x-a|^{s}|y-a|^{t}} d \lambda_{N}(a)
$$


We claim (1) that $G_{N}(x, y)$ is bounded above, independently of $N, x$ and $y$, for all $(x, y)$ in the support of $\mu$ with

$$
(x, y) \notin\left(\{b\} \times R^{d}\right) \cup\left(\mathbb{R}^{d} \times\{b\}\right),
$$

and (2) that $G_{N}(x, y) \rightarrow 0$ pointwise there as $N \uparrow \infty$. Once we have this claim, it will follow from the Lebesgue Dominated Convergence Theorem that $\delta \leq 0$, a contradiction, and the proof will be complete.

To prove the claims, fix $x \neq b$ and $y \neq b$ with $(x, y)$ in the support of $\mu$.

For large $N$, and $a$ belonging to the support of $\lambda_{N}$, we have that $|x-a|$ is comparable to $|x-b|$ and $|y-a|$ is comparable to $|y-b|$, so $G_{N}(x, y)$ is bounded above by twice

$$
\frac{1}{|x-b|^{s} \cdot|y-b|^{t}} \int|a-b|^{s+t} d \lambda_{N}(a) \leq \frac{2^{-(s+t) N}\left\|\lambda_{N}\right\|}{|x-b|^{s} \cdot|y-b|^{t}},
$$

which tends to zero as $N \uparrow \infty$. This proves claim (2).

To get a bound for $G_{n}(x, y)$, choose $p \in \mathbb{N}$ and $q \in \mathbb{N}$ with $x \in A_{p}(b)$ and $y \in A_{q}(b)$.

Fix $n$, with $N \leq n \leq M$, and fix $a$ in the support of $\nu_{n}$.

Consider the following cases, which together cover all possibilities:

(1) $|p-n|>1$ and $|q-n|>1$.

(2) $|p-n|>1$ and $|q-n| \leq 1$.

(3) $|p-n| \leq 1$ and $|q-n|>1$.

(4) $|p-n| \leq 1$ and $|q-n| \leq 1$.

Observe that for given $x$ and $y$, there will be at most 3 values of $n$ for which Case 2 holds, and that the same is true for Cases 3 and 4 .

3.3.1. Case (1)). We have $|x-a| \geq 2^{-(n+2)},|y-a| \geq 2^{-(n+2)},|a-b| \leq$ $2^{-n}$, so

$$
2^{(s+t) n} \int \frac{|a-b|^{s+t}}{|x-a|^{s} \cdot|y-a|^{t}} d \nu_{n}(a) \leq 4^{s+t} \cdot 2^{(s+t) n} \cdot\left\|\nu_{n}\right\| .
$$

3.3.2. Case (2). We have

$$
\begin{aligned}
& 2^{(s+t) n} \int \frac{|a-b|^{s+t}}{|x-a|^{s} \cdot|y-a|^{t}} d \nu_{n}(a) \\
\leq & 4^{s} \cdot \int \frac{d \nu_{n}(a)}{|y-a|^{t}} \\
\leq & 4^{s} \cdot(M+1)^{s} \cdot \int \frac{d \nu_{n}(a)}{|y-a|^{s+t}} \leq 4^{s} \cdot(M+1)^{s},
\end{aligned}
$$

since $|y-a| \leq|y-b|+|b-a| \leq M+1$. 
3.3.3. Case (3). This is similar to Case (2), and we get the bound $4^{t} \cdot(M+1)^{t}$.

3.3.4. Case (4). If $|y-a| \leq|x-a|$, then

$$
\frac{1}{|x-a|^{s} \cdot|y-a|^{t}} \leq \frac{1}{|y-a|^{s+t}}
$$

and if $|x-a| \leq|y-a|$, then

$$
\frac{1}{|x-a|^{s} \cdot|y-a|^{t}} \leq \frac{1}{|x-a|^{s+t}}
$$

so in either case

$$
\frac{1}{|x-a|^{s} \cdot|y-a|^{t}} \leq \max \left\{\frac{1}{|x-a|^{s+t}}, \frac{1}{|y-a|^{s+t}}\right\}
$$

hence

$$
\begin{aligned}
& 2^{(s+t) n} \int \frac{|a-b|^{s+t}}{|x-a|^{s} \cdot|y-a|^{t}} d \nu_{n}(a) \\
\leq & \max \left\{\int \frac{d \nu_{n}(a)}{|x-a|^{s+t}}, \int \frac{d \nu_{n}(a)}{|y-a|^{s+t}}\right\} \leq 1 .
\end{aligned}
$$

Thus, combining all these estimates, we get

$$
\begin{aligned}
G_{N}(x, y) & \leq 4^{s+t} \sum_{N}^{M} 2^{(s+t) n}\left\|\nu_{n}\right\|+6 \cdot(4 M+4)^{\max \{s, t\}}+3 \cdot 1 \\
& \leq 12 \cdot(4 M+4)^{s+t} .
\end{aligned}
$$

This proves claim (1) and concludes the proof of the lemma.

Again we obtain:

Corollary 3.4. Under the same assumptions on $b, \mu, s, \delta$ and $E$, the set $E$ has Lebesgue density zero at $b$.

We expect that similar lemmas could be proved about triple-layer potentials, and so on.

3.4. A Refined Estimate. We can do better if we consider potentials with a factor $|x-y|^{u}$ (with $0<u<1$ ) on the measure:

Lemma 3.5. Let $b \in \mathbb{R}^{d}$, $\mu$ be a positive measure on $\mathbb{R}^{d} \times \mathbb{R}^{d}$ having compact support and no mass on $\{b\} \times \mathbb{R}^{d}$ or on $\mathbb{R}^{d} \times\{b\}$. Let $s>0$, $t>0,0<u<\min \{1, s, t\}$ and $s+t-u<d$. Let $\epsilon>0$, and

$$
E:=\left\{a \in \mathbb{R}^{d}:|a-b|^{s+t-u} \cdot \int_{\mathbb{R}^{d} \times \mathbb{R}^{d}} \frac{|x-y|^{u} d \mu(x, y)}{|x-a|^{s}|y-a|^{t}} \geq \epsilon\right\} .
$$


Then

$$
\sum_{n=1}^{\infty} 2^{(s+t-u) n} C_{s+t-u}\left(A_{n}(b) \cap E\right)<+\infty .
$$

Proof. Since $0<u<1$, we have $|x-y|^{u} \leq|x-a|^{u}+|y-a|^{u}$, so

$$
\frac{|x-y|^{u}}{|x-a|^{s}|y-a|^{t}} \leq \frac{1}{|x-a|^{s-u}|y-a|^{t}}+\frac{1}{|x-a|^{s}|y-a|^{t-u}} .
$$

Thus $E_{1} \cap E_{2} \subset E$, where

$$
E_{1}:=\left\{a \in \mathbb{R}^{d}:|a-b|^{s+t-u} \cdot \int_{\mathbb{R}^{d} \times \mathbb{R}^{d}} \frac{d \mu(x, y)}{|x-a|^{s-u}|y-a|^{t}} \geq \epsilon / 2\right\}
$$

and

$$
E_{2}:=\left\{a \in \mathbb{R}^{d}:|a-b|^{s+t-u} \cdot \int_{\mathbb{R}^{d} \times \mathbb{R}^{d}} \frac{d \mu(x, y)}{|x-a|^{s}|y-a|^{t-u}} \geq \epsilon / 2\right\} .
$$

Applying Lemma 3.3 twice, we see that $E_{1}$ and $E_{2}$ satisfy

$$
\sum_{n=1}^{\infty} 2^{(s+t-u) n} C_{s+t-u}\left(A_{n}(b) \cap E_{j}\right)<+\infty,
$$

so the result follows from the subadditivity of $C_{s+t-u}$.

Again we obtain:

Corollary 3.6. Under the same assumptions on $b, \mu, s, \delta$ and $E$, the set $E$ has Lebesgue density zero at $b$.

3.5. A Density Lemma. In the next lemma, we remove a restriction on $\mu$ : the measure is allowed to have mass on the horizontal and vertical slices through the point $b$, as long as it has no mass at the point $(b, b)$. The conclusion is not the convergence of a Wiener-type series, but that an exceptional set has Lebesgue density zero.

Lemma 3.7. Let $b \in \mathbb{R}^{d}$, and $\mu$ be a positive measure on $\mathbb{R}^{d} \times \mathbb{R}^{d}$ having compact support and no mass at the point $(b, b)$. Let $s>0$, $t>0,0<u<\min \{1, s, t\}$ and $s+t-u<d$. Let $\epsilon>0$, and

$$
E:=\left\{a \in \mathbb{R}^{d}:|a-b|^{s+t-u} \cdot \int_{\mathbb{R}^{d} \times \mathbb{R}^{d}} \frac{|x-y|^{u} d \mu(x, y)}{|x-a|^{s}|y-a|^{t}} \geq \epsilon\right\} .
$$

Then E has Lebesgue density zero at $b$.

For convenience, let us say that a set $E$ is $s$-thin $a t b$ if

$$
\sum_{n=1}^{\infty} 2^{s n} C_{s}\left(A_{n}(b) \cap E\right)<+\infty .
$$


As mentioned already, if $E$ is $s$-thin at $b$ for some $s$ with $0<s<d$, then $E$ has Lebesgue density zero at $b$. So if $E \subset E_{1} \cup \cdots \cup E_{n}$ and for each $j$ there is some $s_{j} \in(0, d)$ such that $E_{j}$ is $s_{j}$-thin, then $E$ has Lebesgue density zero at $b$.

Proof. Let us denote the double-layer potential in the statement by $P(\mu)$ :

$$
P(\mu)(a):=\int_{\mathbb{R}^{d} \times \mathbb{R}^{d}} \frac{|x-y|^{u} d \mu(x, y)}{|x-a|^{s}|y-a|^{t}} .
$$

Write $\mu=\mu_{1}+\mu_{2}+\mu_{3}$, where $\mu_{2}$ is the restriction of $\mu$ to $V:=\{b\} \times \mathbb{R}^{d}$ (i.e. $\mu_{2}(T)=\mu(T \cap V)$ for all sets $\left.T\right), \mu_{3}$ is the restriction of $\mu$ to $H:=\mathbb{R}^{d} \times\{b\}$, and $\mu_{1}$ is the restriction of $\mu$ to the complement of $H \cup V$. Then we may write $P(\mu)=P_{1}(a)+P_{2}(a)+P_{3}(a)$, where $P_{j}:=P\left(\mu_{j}\right)$.

Note that none of the $\mu_{j}$ has a point mass at $(b, b)$ and that $\mu_{1}$ has no mass on $V \cup H$.

Let

$$
E_{j}:=\left\{a:|a-b|^{s+t-u} \cdot P_{j}(a) \geq \delta / 3\right\}
$$

for $j=1,2,3$. Then $E \subset E_{1} \cup E_{2} \cup E_{3}$, so it suffices to show that each $E_{j}$ has Lebesgue density zero at $b$.

Lemma 3.5 applies, with $\mu$ replaced by $\mu_{1}$, and tells us that $E_{1}$ is $(s+t-u)$-thin at $b$, so the case $j=1$ is done.

Consider the case $j=2$.

Using the fact that $t \mapsto t^{u}$ is subadditive, as in the proof of Lemma 3.5, we see that $P_{2}(a) \leq P_{21}(a)+P_{22}(a)$, where

$$
\begin{aligned}
& P_{21}(a)=\int \frac{d \mu(x, y)}{|x-a|^{s-u} \cdot|y-a|^{t}} \\
& P_{22}(a)=\int \frac{d \mu(x, y)}{|x-a|^{s} \cdot|y-a|^{t-u}} .
\end{aligned}
$$

Let

$$
E_{2 k}:=\left\{a:|a-b|^{s+t-u} \cdot P_{2 k}(a) \geq \delta / 6\right\}
$$

for $k=1,2$. Then $E_{2} \subset E_{21} \cup E_{22}$, so it suffices to show that both $E_{2 k}$ have Lebesgue density zero at $b$.

Consider $E_{21}$. The measure $\mu_{2}$ is supported on $V=\{b\} \times \mathbb{R}^{d}$, so it is the product of the unit point mass at $b$ and a measure $\mu_{22}$ on $\mathbb{R}^{d}$, and we have

$$
P_{21}(a)=\frac{1}{|b-a|^{s-u}} \cdot \int_{\mathbb{R}^{d}} \frac{d \mu_{22}(y)}{|y-a|^{t}},
$$


SO

$$
E_{21}=\left\{a:|a-b|^{t} \cdot \int_{\mathbb{R}^{d}} \frac{d \mu_{22}(y)}{|y-a|^{t}} \geq \delta / 6\right\} .
$$

Since $\mu_{2}$ has no point mass at $(b, b)$, it follows that $\mu_{21}$ has no point mass at $b$. Thus we may apply Lemma 3.1. with $s$ replaced by $t$, to conclude that $E_{21}$ is $t$-thin at $b$.

Next, consider $E_{22}$. We have

$$
P_{22}(a)=\frac{1}{|b-a|^{s}} \cdot \int_{\mathbb{R}^{d}} \frac{d \mu_{22}(y)}{|y-a|^{t-u}},
$$

So

$$
E_{22}=\left\{a:|a-b|^{t-u} \cdot \int_{\mathbb{R}^{d}} \frac{d \mu_{22}(y)}{|y-a|^{t-u}} \geq \delta / 6\right\} .
$$

So we may apply Lemma 3.1, with $s$ replaced by $t-u$, to conclude that $E_{22}$ is $(t-u)$-thin at $b$.

Thus $E_{2}$ has density zero at $b$.

A completely parallel argument shows that $E_{3}$ is contained in the union of an $s$-thin set and an $(s-u)$-thin set, and hence has density zero at $b$.

The proof is complete.

The above argument does not work if we try to relax the condition $u<\min \{1, s, t\}$. In the sequel we shall encounter a case with $d=2$ and $s=t=u=1$, so we record a substitute result:

Lemma 3.8. Let $b \in \mathbb{R}^{d}$, and $\mu$ be a positive measure on $\mathbb{R}^{d} \times \mathbb{R}^{d}$ having compact support and no mass at the point $(b, b)$. Let $s>0$, $t>0,0<u \leq \min \{1, s, t\}$ and $s+t-u<d$. Then there exists a positive constant $M$ such that the set

$$
E:=\left\{a \in \mathbb{R}^{d}:|a-b|^{s+t-u} \cdot \int_{\mathbb{R}^{d} \times \mathbb{R}^{d}} \frac{|x-y|^{u} d \mu(x, y)}{|x-a|^{s}|y-a|^{t}} \geq M\right\} .
$$

has Lebesgue density zero at $b$.

Proof. It remains only to consider the cases $u=1, u=s$ and $u=t$.

We can follow the same proof as in Lemma 3.7, with $\delta$ replaced by $M$, and most of it works for any positive $M$.

The case $u=1$ causes no problem.

When $u=s$ or $u=t$ and we consider $E_{1}$, we may have to apply Lemma 3.1 instead of Lemma 3.3, as the integrand depends on only one of the variables $x$ and $y$. 
When we come to consider $E_{22}$, and now suppose that $u=t$, we find that

$$
P_{22}(a)=\frac{1}{|b-a|^{s}} \cdot\|\mu\|
$$

SO

$$
E_{22}=\{a:\|\mu\| \geq M / 6\} .
$$

Thus $E_{22}$ is either empty or $\mathbb{R}^{d}$, depending on the value of $M$. If we take $M=6\|\mu\|$ (or greater), then $E_{22}=\emptyset$, which surely has density zero at $b$.

\section{Dual Spaces and Distributions}

Recall our standing assumptions from Subsection 2.1. In particular, throughout this section we use the facts that $0<\alpha<1, X$ is a compact subset of $\mathbb{C}$ and $b \in X$.

4.1. A Construction. If $\mu$ is any complex measure on $X \times X$, having no mass on the diagonal, we define

$$
L(\mu)(f):=\int_{X \times X} \frac{f(z)-f(w)}{|z-w|^{\alpha}} d \mu(z, w), \forall f \in \operatorname{lip} \alpha .
$$

Then $L(\mu)$ is an element of $(\operatorname{lip} \alpha)^{*}$, the dual of lip $\alpha$, of norm at most $\|\mu\|$. (This construction is classical.)

The linear map $\mu \mapsto L(\mu)$ is not injective, because of the antisymmetry in the integrand. If we define $R(z, w):=(w, z)$, then $L\left(R_{\sharp} \mu\right)=$ $-L(\mu)$, where $R_{\sharp} \mu$ is the push-forward measure, defined by

$$
R_{\sharp} \mu(E)=\mu\left(R^{-1}(E)\right), \forall E \subset X \times X .
$$

We record here, for future use, a consequence of this remark:

Lemma 4.1. Let $b \in X$. Let $\mu$ be a complex measure on $X \times X$, having no mass on the diagonal. Then there exists a measure $\mu^{\prime}$ on $X \times X$ such that $\mu^{\prime}$ has no mass on the vertical slice $V=\{b\} \times X$ and $L(\mu)=L\left(\mu^{\prime}\right)$.

Proof. Write $\mu=\mu_{1}+\mu_{2}$, where $\mu_{2}$ is the restriction of $\mu$ to $V$. Then $\mu_{1}$ has no mass on $V$, and $\mu_{2}$ has no mass at $(b, b)$. Take $\mu^{\prime}=\mu_{1}-R_{\sharp} \mu_{2}$. Then since $R_{\sharp} \mu_{2}$ is supported on the horizontal slice $H=X \times\{b\}$ and has no mass at $(b, b)$, it has no mass on $V$. Also, $L\left(\mu^{\prime}\right)=L(\mu)$. 
4.2. Distributions. Let $\mathcal{D}$ denote the space of test functions (i.e. $C^{\infty}$ functions having compact support), and let $\mathcal{D}^{\prime}$ denote its dual, the Schwartz distribution space.

The restriction of $L(\mu)$ to $\mathcal{D}$ is a distribution. We denote it by the same symbol $L=L(\mu)$. This distribution will not, in general, be representable by integration against a locally-integrable function or a measure.

Since $\langle\phi, L\rangle$ is unaffected if $\phi$ is altered away from $X$, it is clear that $L$ has support in $X$. Thus we can also define $\langle\phi, L\rangle$ for any function $\phi$ defined and $C^{\infty}$ on a neighbourhood of $X$ to be $\langle\tilde{\phi}, L\rangle$ where $\tilde{\phi}$ is any element of $\mathcal{E}$ (the space of globally-defined $C^{\infty}$ functions) that agrees with $\phi$ near $X$. For instance, $\left\langle\frac{1}{z-a}, L\right\rangle$ makes sense, for $a \notin X$. Similarly, $\langle f, L\rangle$ makes sense whenever $f$ is defined on some neighbourhood of $X$ and satisfies a little-lip $\alpha$ condition there.

4.3. Cauchy Transforms. The Cauchy transform of $\phi \in \mathcal{D}$ is its convolution

$$
\hat{\phi}:=\phi *\left(\frac{1}{\pi z}\right)
$$

with the fundamental solution of $\frac{\partial}{\partial \bar{z}}$. In other words,

$$
\hat{\phi}(z)=\frac{1}{\pi} \int \frac{\phi(\zeta)}{z-\zeta} d \mathcal{L}^{2}(\zeta)
$$

for all $z \in \mathbb{C}$. This function belongs to the space $\mathcal{E}$, and satisfies

$$
\frac{\partial \hat{\phi}}{\partial z}=\phi
$$

For distributions $T$ having compact support, we define

$$
\langle\phi, \hat{T}\rangle=-\langle\hat{\phi}, T\rangle, \forall \phi \in \mathcal{D} \text {. }
$$

To analyse the transform in case $T=L(\mu)$, consider the function

$$
H(a):=H(\mu)(a):=\frac{1}{\pi} \int \frac{z-w}{(z-a)(w-a)|z-w|^{\alpha}} d \mu(z, w),
$$

for $a \in \mathbb{C}$. This is well-defined whenever

$$
\widetilde{H}(a):=\widetilde{H}(\mu)(a):=\int \frac{|z-w|^{1-\alpha}}{|z-a| \cdot|w-a|} d|\mu|(z, w)<\infty,
$$

which happens almost everywhere with respect to area measure, and $\tilde{H}$ is locally-integrable, as is seen by an application of Fubini's Theorem. 
Also $|H(a)| \leq \tilde{H}(a)$ for all such $a$. Another Fubini calculation yields

$$
\langle\phi, \widehat{L(\mu)}\rangle=\int_{\mathbb{C}} \phi \cdot H d \mathcal{L}^{2},
$$

for all $\phi \in \mathcal{D}$. Thus $H$ represents $\widehat{L}$. Based on this, we usually write $\widehat{L}(a)$ for $H(a)$. Note that

$$
\widehat{L}(a)=H(a)=\left\langle\frac{1}{\pi(a-z)}, L\right\rangle,
$$

whenever $a \notin X$.

4.4. Estimates for the Cauchy Transform. First, an estimate for $\widetilde{H}(\mu)$. Notice that $\widetilde{H}(\mu)$ depends only on the total variation measure $|\mu|$.

Lemma 4.2. Let the complex measure $\mu$ have support in $X \times X$ and no mass at the point $(b, b)$. Let $\delta>0$ be given. Then the set

$$
E:=\left\{a:|a-b|^{1+\alpha} \cdot \widetilde{H}(\mu)(a)<\delta\right\}
$$

has full area density at $b$.

Proof. Just apply Lemma 3.7 with $d=2, \mu=|\mu|, s=t=1$ and $u=1-\alpha$.

Next, an estimate for $\widetilde{H}\left(|z-w|^{\alpha} \cdot \mu\right.$ ) (by $|z-w|^{\alpha} \cdot \mu$ we mean the measure obtained by multiplying $\mu$ by the function $\left.(z, w) \mapsto|z-w|^{\alpha}\right)$.

Lemma 4.3. Let the complex measure $\mu$ have support in $X \times X$ and no mass at the point $(b, b)$. Let $\delta>0$ be given. Then there exists a constant $M>0$ such that the set

$$
E:=\left\{a:|a-b| \cdot \widetilde{H}\left(|z-w|^{\alpha} \cdot \mu\right)(a)<M\right\}
$$

has full area density at $b$.

Proof. Explicitly,

$$
\widetilde{H}\left(|z-w|^{\alpha} \cdot \mu\right)(a)=\int_{X \times X} \frac{|z-w| d|\mu|(z, w)}{|z-a| \cdot|w-a|}
$$

so we use the case of Lemma 3.8 with $d=2, \mu=|\mu|, s=t=1$ and $u=1$.

Next, a uniform estimate for the Cauchy transforms of all bounded multiples of a fixed measure $\mu$ : 
Lemma 4.4. Let the complex measure $\mu$ have compact support in $\mathbb{C} \times \mathbb{C}$ and no mass at the point $(b, b)$. Let $\delta>0$. Then there exists a set $E$ having full area density at $b$ such that

$$
|a-b|^{1+\alpha} \cdot|\widehat{L(f \cdot \mu)}(a)|<\delta \cdot \sup |f|
$$

for all $a \in E$, whenever $f: X \times X \rightarrow \mathbb{C}$ is a bounded Borel function.

Proof. Take the set $E$ given by Lemma 4.2, Then the desired estimate holds on $E$ since

$$
|\widehat{L(f \cdot \mu)}(a)| \leq \widetilde{H}(\mu)(a) \cdot \sup |f|
$$

for all $a$ for which the right-hand-side is finite.

4.5. The Product $g \cdot L$. The dual of any Banach algebra is naturally a module over the algebra. In the present situation, lip $\alpha$ acts on $(\operatorname{lip} \alpha)^{*}$, so given $g \in \operatorname{lip} \alpha$ and a measure $\mu$ on $X \times X$, we may define a new element $g \cdot L(\mu)$ of $(\operatorname{lip} \alpha)^{*}$ by setting

$$
\langle f, g \cdot L\rangle=\langle g \cdot f, L\rangle, \forall f \in \operatorname{lip} \alpha .
$$

We remark that $\langle 1, g \cdot L\rangle=\langle g, L\rangle \neq 0$, in general, so we cannot represent $g \cdot L$ by a measure as in Equation (11). However, writing

$$
f(z) g(z)-f(w) g(w)=(f(z)-f(w)) \cdot g(z)+f(w) \cdot(g(z)-g(w)),
$$

a short calculation gives

$$
\langle f, g \cdot L\rangle=\int_{X \times X} \frac{f(z)-f(w)}{|z-w|^{\alpha}} d \nu(z, w)+\int_{X} f(w) d \lambda(w),
$$

where $\nu=\left(g \circ \pi_{1}\right) \cdot \mu$ is the measure on $X \times X$ such that

$$
\nu(E)=\int_{E} g(z) d \mu(z, w)
$$

whenever $E \subset X \times X$ is a Borel set, and $\lambda$ is the measure on $X$ such that

$$
\lambda(E)=\int_{E \times X} \frac{g(z)-g(w)}{|z-w|^{\alpha}} d \mu(z, w)
$$

whenever $E \subset X$ is Borel, i.e. $\lambda$ is the first-coordinate marginal of the measure

$$
\frac{g(z)-g(w)}{|z-w|^{\alpha}} \cdot \mu(z, w)
$$

(a bounded multiple of $\mu$ ). So we may write $g \cdot L(\mu)=S_{1}+S_{2}$, where $S_{1}=L(\nu)$, and $S_{2}$ is represented by the measure $\lambda$ on $X$.

Let us call $S_{1}$ the main part of $g \cdot L$ and $S_{2}$ the residual part of $g \cdot L$. 
We note for future reference that the measures $\nu$ and $\lambda$ have totalvariation measures dominated by fixed measures depending only on $\mu$ and on the lip $\alpha$ norm $\|g\|=\|g\|_{\alpha}=\|g\|^{\prime}+\sup |g|$ of $g$ :

Lemma 4.5. Let $\mu$ be a measure on $X \times X$ having no mass on the diagonal. Let $\mu_{\sharp}$ be the first-coordinate marginal of $|\mu|$. Then for any $g \in \operatorname{lip} \alpha$, the measures $\nu$ and $\lambda$ representing the main and residual parts of $g \cdot L(\mu)$ satisfy

(1) $|\nu| \leq|g(z) \cdot \mu|$, and (2) $|\lambda| \leq\|g\|^{\prime} \cdot\left|\mu_{\sharp}\right|$.

4.6. Estimate for the Product. We now establish a lemma that gives a set having full area density on which a uniform estimate holds for $\widehat{g \cdot L(\mu})$, provided $g(b)=0$.

Lemma 4.6. Let $0<\alpha<1$, and $b \in X$, and let the complex measure $\mu$ have support in $X \times X$ and no mass on the diagonal. Then there exist a constant $K>0$ and a set $E \subset \mathbb{C}$ having full area density at $b$, such that whenever $g \in \tilde{A}$, with $g(b)=0$, we have

$$
|a-b| \cdot|\widehat{g \cdot L(\mu)}(a)| \leq K \cdot\|g\|_{\alpha}
$$

for all $a \in E$.

Proof. By Lemma 4.1, we may assume that $\mu$ has no mass on the vertical slice $V=\{b\} \times X$, since we can if necessary replace it, without altering $L(\mu)$, by another measure $\mu^{\prime}$ having no mass on $V$.

Also, by Corollary 3.2 (taking $\delta=1$ ), there is a set $E_{1}$ having full density at $b$, such that

$$
|a-b| \cdot \int_{\mathbb{R}^{d}} \frac{d\left|\mu_{\sharp}\right|(z)}{|z-a|} \leq 1
$$

for all $a \in E_{1}$, where $\mu_{\sharp}$ is the usual marginal (since $\mu_{\sharp}$ has no mass at $b)$.

Applying Lemma 4.2 with $\delta=1$, there is a set $E_{2}$ having full density at $b$, such that

for all $a \in E_{2}$.

$$
|a-b|^{1+\alpha} \cdot|\widehat{H}(\mu)(a)| \leq 1
$$

By Lemma 4.3 there is a constant $M>0$ such that

$$
|a-b| \cdot\left|\widetilde{H}\left(|z-w|^{\alpha} \cdot \mu\right)(a)\right| \leq M
$$

for all $a \in E_{3}$, a set having full density at $b$.

Take $E=E_{1} \cap E_{2} \cap E_{3}$. Then $E$ has full density at $b$. 
Fix any $g \in \operatorname{lip} \alpha$, with $g(b)=0$ and $\|g\| \leq 1$, and take $a \in E$. Let $S_{1}=L(\nu)$ and $S_{2}=\lambda$ be the main and residual parts of $g \cdot L(\mu)$. By Lemma 4.5, $|\nu| \leq|g(z) \cdot \mu| \leq|z-b|^{\alpha} \cdot|\mu| \leq|z-a|^{\alpha} \cdot|\mu|+|a-b|^{\alpha} \cdot|\mu|$, and $|\lambda| \leq \mu_{\sharp}$, and we have

$$
\widehat{g \cdot L(\mu)}=\widehat{L(\nu)}+\widehat{\lambda}
$$

so for all $a \in E$

$$
\begin{aligned}
& |\widehat{g \cdot L(\mu)}(a)| \\
\leq & \widetilde{H}(\nu)(a)+\int_{\mathbb{C}} \frac{d|\lambda|(z)}{|z-a|} \\
\leq & \widetilde{H}\left(|z-b|^{\alpha} \cdot|\mu|\right)(a)+|a-b|^{\alpha} \cdot \widetilde{H}(\mu)(a)+\int \frac{d\left|\mu_{\sharp}\right|(z)}{|z-a|}
\end{aligned}
$$

SO

$$
|a-b| \cdot|\widehat{g \cdot L(\mu)}(a)| \leq M+2
$$

Taking $K=M+2$, we are done.

\section{Proof of TheOrem}

Suppose $A=A_{\alpha}(U)$ admits a nonzero continuous point derivation at $b \in X=\operatorname{bdy}(U)$, and let $\partial$ be the normalized derivation at that point.

There is a complex measure $\mu$ on $X \times X$ having no mass on the diagonal, such that $\partial f=L(\mu)(f)$ whenever $f \in \tilde{A}$. (We express this by saying that the distribution $T_{1}:=L(\mu)$ is continuous on lip $\alpha$ and represents the derivation on $\tilde{A}$.) This fact was explained in our previous paper [11, pp. 135-6, or p. 6 in the ArXiv copy].

By Lemmas 4.4 and 4.6 there exist a constant $K>0$ and a set $E$ having full area density at $b$, such that for all $a \in E$ we have

$$
\begin{aligned}
|a-b|^{1+\alpha} \cdot\left|\widehat{T}_{1}(a)\right| & \leq 1, \\
|a-b| \cdot \frac{g}{g \cdot T_{1}}(a) & \leq K .
\end{aligned}
$$

Since $U$ has full density at $b$, we may (and we do) assume $E \subset U$, by taking the intersection if need be.

Defining distributions (and elements of lip $\left.\alpha^{*}\right) T_{0}:=(z-b) \cdot T_{1}$, and $T:=-\pi(z-b)^{2} \cdot T_{1}$, one sees that $T_{0}(f)=f(b)$ and $T(f)=0$ for all $f \in \mathcal{A}$, and hence for all $f \in \tilde{A}$ by continuity. In other words, $T_{0}$ represents evaluation at $b$ on $\tilde{A}$ and $T=-\pi(z-b) T_{0}$ annihilates $\tilde{A}$.

All these distributions have compact support (on $X$ ) and so have Cauchy transforms, which are holomorphic when restricted to $U$. 
Next (see [11] for details),

$$
\widehat{T}_{0}=(z-b) \cdot \widehat{T}_{1},
$$

and

$$
\widehat{T}=1-\pi(z-b)^{2} \cdot \widehat{T}_{1}
$$

Thus, for $a \in E$, we have

$$
|\widehat{T}(a)-1| \leq|a-b|^{1-\alpha}
$$

(Note that the exponent $1-\alpha$ was mistakenly entered as $\alpha$ in [11, Equation (3), page 142]. The ArXiv version is corrected.) So, replacing $E$, if need be, by its intersection with the open ball of radius 1 about $b$, we may assume that $\widehat{T}(a) \neq 0$ on $E$.

Then whenever $a \in E$ we may form the distribution

$$
R_{a}:=\frac{1}{\pi \widehat{T}(a)(a-z)} \cdot T,
$$

Then $R_{a}$ represents evaluation at $a$ on $A$, and the functional

$$
f \mapsto \frac{f(a)-f(b)}{a-b}-\partial f
$$

is represented on $A$ by the distribution

$$
D_{a}=\frac{R_{a}-T_{0}}{a-b}-T_{1}
$$

Then $D_{a} f \rightarrow 0$ for all $f \in \mathcal{A}$ as $a \rightarrow b$, with $a \in E$. To prove the theorem, we have to show that this also holds for all $f$ in the closure $\tilde{A}$ of $\mathcal{A}$. To do this, it suffices to show that the functionals $D_{a}$ are uniformly bounded on $\tilde{A}$, for $a \in E$, i.e that

$$
\left|D_{a}(f)\right| \leq c\|f\|_{\alpha}
$$

for some constant $c>0$, for all $f \in A$ and all $a \in E$.

Fix an arbitrary $f \in \operatorname{lip} \alpha$, holomorphic on $U$, with $\|f\|_{\alpha} \leq 1$. Take $g(z)=f(z)-f(b)$, so $D_{a}(f)=D_{a}(g),\|f\|^{\prime}=\|g\|^{\prime}$ and $g(\bar{b})=0$, so that $|g(a)| \leq|a-b|^{\alpha}$. A calculation shows that

$$
D_{a}(g)=(a-b) \cdot \widehat{g \cdot T_{1}}(a)+\pi(a-b) g(a) \widehat{T}_{1}(a) .
$$

Thus, for $a \in E$, we have

$$
\left|D_{a}(g)\right| \leq|a-b| \cdot\left|\widehat{g \cdot T_{1}}(a)\right|+\pi|a-b|^{1+\alpha} \cdot\left|\widehat{T}_{1}(a)\right| \leq K+\pi .
$$

Thus $D_{a}(f)$ is indeed bounded, as required. This concludes the proof. 


\section{REMARKS}

6.1. The set $E$ constructed in the proof has "more than" full area density. To be precise, the complement of $E$ is a finite union of exceptional sets $E^{\prime}$ for which one of the conditions

- $\sum_{n} 2^{n} C_{1}\left(A_{n}(b) \cap E^{\prime}\right)<+\infty$,

- $\sum_{n} 2^{\alpha n} C_{\alpha}\left(A_{n}(b) \cap E^{\prime}\right)<+\infty$, or

- $\sum_{n} 2^{(1+\alpha) n} C_{1+\alpha}\left(A_{n}(b) \cap E^{\prime}\right)<+\infty$

holds. The weakest of these conditions is the latter, so we can say that

$$
\sum_{n} 2^{(1+\alpha) n} C_{1+\alpha}\left(A_{n}(b) \backslash E\right)<+\infty .
$$

The convergence of this Wiener-type series implies that the $(1+\alpha)$ dimensional density of $C_{1+\alpha}$ capacity

$$
\lim _{r \downarrow 0} \frac{C_{1+\alpha}(\mathbb{B}(b, r) \backslash E)}{r^{1+\alpha}}=0,
$$

and, a fortiori, the $\beta$-dimensional density of $\beta$-dimensional Hausdorff content

$$
\lim _{r \downarrow 0} \frac{M^{\beta}(\mathbb{B}(b, r) \backslash E)}{r^{\beta}}=0
$$

for $1+\alpha<\beta \leq 2$.

6.2. It would be interesting to know whether the conditions of Corollary 1.3 are equivalent to the existence of a sequence $a_{n} \rightarrow b$ along which $f^{\prime}\left(a_{n}\right)$ is bounded, for each $f \in A_{\alpha}(U)$.

6.3. I am grateful to David Malone and Oliver Mason, and to the referees for helpful comment on this paper.

\section{REFERENCES}

1. A. Browder. Introduction to Function Algebras. Benjamin. New York. 1969.

2. E.P. Dolzhenko. On removal of singularities of analytic functions. Uspehi Mat. Nauk 18, no.4 (112) (1963) 135-42. English transl., AMS Translations (2) 97 (1971) 33-41.

3. H. Federer. Geometric Measure Theory. Springer. New York. 1969.

4. D. Armitage and S. Gardiner. Classical Potential Theory. Springer.New York. 2001.

5. L. Hormander. The Analysis of Linear Partial Differential Operators I. Springer. New York. 1983.

6. D. Lord and A.G. O'Farrell. J. d'Analyse Math. (Jerusalem) 63 (1994) 103-19.

7. Y. Mizuta. Potential Theory in Euclidean Spaces. Gakkōtosho. Tokyo. 1996. 
8. A.G. O'Farrell. Equiconvergence of Derivations. Pacific J. Math. 53 (1974) 53954.

9. A.G. O'Farrell. Analytic capacity, Hölder conditions, and $\tau$-spikes. Transaction AMS 196 (1974) 415-24.

10. A.G. O'Farrell. Annihilators of Rational Modules. J. Functional Analysis 19 (1975) 373-89.

11. A.G. O'Farrell. Boundary smoothness of analytic functions. Analysis and Mathematical Physics, 4 (2014) 131-44.

12. L. Schwartz. Théorie des distributions. 2nd ed. Hermann. Paris. 1966.

13. D.R. Sherbert. The structure of ideals and point derivations in Banach algebras of Lipschitz functions. Transactions AMS 111(1964) 240-72.

E-mail address: anthony.ofarrell@nuim.ie

Mathematics and Statistics, NUi, Maynooth, Co. Kildare, Ireland 